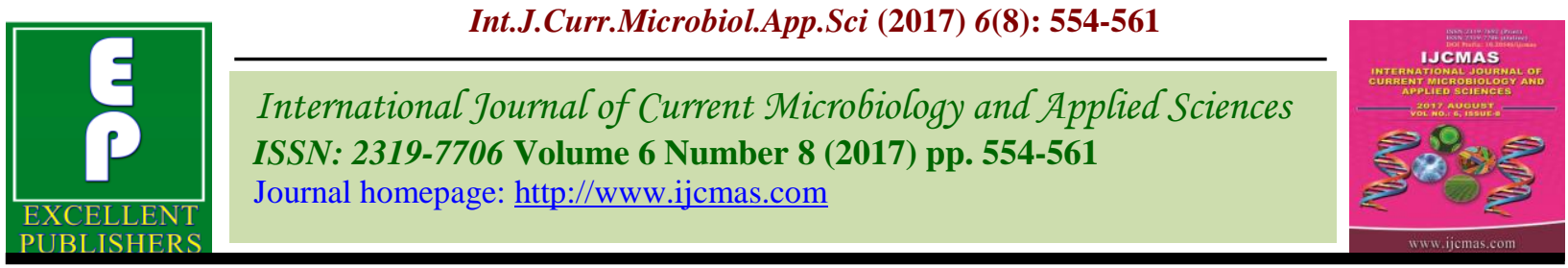

Original Research Article

https://doi.org/10.20546/ijcmas.2017.608.072

\title{
Combining Ability for Grain Yield and its Components Involving Alloplasmic Iso-Nuclear Lines of Pearl Millet
}

\author{
Anil Kumar*, R. Kumar, Devvart and A.K. Dehinwal \\ Department of Genetics and Plant Breeding, CCS Haryana Agricultural University, \\ Hisar-125 004, Haryana, India \\ *Corresponding author
}

A B S T R A C T

\begin{tabular}{|l|}
\hline Ke y w or d s \\
Pearl millet, \\
Combining \\
ability, Diverse \\
CMS Sources, \\
Line x tester \\
analysis.
\end{tabular}

Combining ability for grain yield and its components in pearl millet was studied in 48 hybrids developed by crossing six A- and their six B-lines with $8 \mathrm{R}$-lines in a line $\mathrm{x}$ tester mating design. The three A- lines viz. $81 \mathrm{~A}_{1}, 81 \mathrm{~A}_{4}$ and $81 \mathrm{~A}_{5}$ and their corresponding $\mathrm{B}$ - lines represented three different systems of male sterility and were very diverse. The 12 parents and the 48 hybrids were grown separately in contiguous blocks in $2 \mathrm{R} \times 4.0 \mathrm{~m} \times 0.45 \mathrm{~m}$ in randomized block design with three replications in two environments viz., normal sown and late sown condition at Research Farm, CCS Haryana Agricultural University, Hisar during 2013. The analysis of variance for combining ability revealed that the mean squares due to lines, testers and lines $\times$ testers were significant for all the characters in both the environments except mean squares due to lines for grain yield in $\mathrm{E}_{2}$, days to $50 \%$ flowering in $\mathrm{E}_{2}$, panicle girth in both environments and harvest index in $\mathrm{E}_{1}$. The magnitude of sca variances exceeded to that of gca variances for all the characters viz. grain yield per plant, dry fodder yield per plant, days to 50 per cent flowering, plant height, effective tillers, 1000-seed weight, panicle girth, panicle length and harvest index, indicated preponderance of non additive type of gene action in the expression of these traits. None of the parents- lines (sterile as well as maintainer) and tester proved to be good general combiner for all the characters and across the environments. The results indicated that line $1\left(81 \mathrm{~A}_{1}\right)$ representing $\mathrm{A}_{1}$ male sterile cytoplasm proved to be good general combiner for earliness, panicle length and harvest index in one of the two environments and $4\left(81 \mathrm{~B}_{1}\right)$ representing $\mathrm{A}_{1}$ system fertile cytoplasm (maintainer) combined favorably for grain yield, dry fodder yield and effective tillers. The combining ability of the lines $1 / 4$ $\left(81 \mathrm{~A}_{1} / 81 \mathrm{~B}_{1}\right)$ and $2 / 5\left(81 \mathrm{~A}_{4} / 81 \mathrm{~B}_{4}\right)$ was not similar at CMS system level. Line $2\left(81 \mathrm{~A}_{4}\right)$ combined better for effective tillers and line $5\left(81 \mathrm{~B}_{4}\right)$ for plant height, dry fodder yield and 1000 -grain weight. The line $6\left(81 \mathrm{~B}_{5}\right)$ combined better for plant height only. The tester 14 (HTP 92/80) turned to be good general combiner in one or both the environments for grain yield and some other traits such as dry fodder yield, earliness and panicle length. The hybrid $3 \times 11\left(81 \mathrm{~A}_{5} \times \mathrm{ERC} / 99 / 35\right)$ expressed significant positive sca effects in one of the two environments for grain yield major component traits such as effective tillers, panicle girth and harvest index (positive sca effects), therefore, this hybrid needs further vigorous testing. The crosses involving ERC/99/35, MRC/10/61, H77/29-2 and TCH 26-1 were found to be highly heterotic.

\section{Introduction}

Pearl millet [Pennisetum glaucum (L.) R. Br.] is an important cereal crop grown in tropical semi-arid regions of the world primarily in Africa and Asia. It is primarily grown for grain production, but also valued for its fodder (both stover and green forage). It is nutritionally very rich, supplying highest number of calories than all other cereals, having high protein, fibre and fat content (NIN, 2003). It combats the life style diseases 
especially diabetes and cardiovascular if consumed properly and regularly. It is highly cross pollinated crop, grown over $7.29 \mathrm{~m}$ ha with grain production of $8.74 \mathrm{~m}$ tonnes and productivity of $1198 \mathrm{~kg} / \mathrm{ha}$ in India (Anonymous, 2016).

Hybrid cultivar development in pearl millet became possible with the discovery of cytoplasmic- genic male-sterility (Burton, 1958). The use of CMS (cytoplasmic-genic male sterility) in pearl millet paved the way for grain yield augmentation with the development and release of first grain hybrid HB-I (Tift 23 A x BIL-3B) by Athwal (1965). Since then, large number of hybrids have been developed and commercialized in India, largely based on the $\mathrm{A}_{1} \mathrm{CMS}$ system.

However, several other sources of malesterility inducing cytoplasms besides $\mathrm{A}_{1}$ (Burton, 1965) such as $\mathrm{A}_{2}, \mathrm{~A}_{3}$ (Burton and Athwal, 1967), PT732A (Appadurai et al., 1982), ex-Bornu (Gero) (Aken'ova, 1985), violaceum (Marchais and Pernes, 1985), $\mathrm{A}_{4}$ (Hanna, 1989) and $A_{5}$ (Rai, 1995) have been reported to diversify the cytoplasm and nuclear genetic base of pearl millet hybrids. However, commercial utilization of different CMS systems depends on several factors including their effect on agronomic traits. The iso-nuclear lines have also been established in the background of several diverse CMS sources, which provides an opportunity for studying cytoplasmic effects on the expression of different characters. The information on combining ability of lines is helpful in precise estimate of genetic parameters, the knowledge about nicking ability of lines and determining relative chance of heterosis in hybrids carrying different cytoplasm in pearl millet. Studies have indicated that cytoplasm exhibits pronounced effect on combining ability (Young and Virmani, 1990). Therefore, the investigation was undertaken to study the combining ability for grain yield and its component characters involving alloplasmic iso-nuclear lines of pearl millet.

\section{Materials and Methods}

The material for present study consisted of three CMS lines (A-lines) namely $81 \mathrm{~A}_{1}, 81 \mathrm{~A}_{4}$ and $81 \mathrm{~A}_{5}$ their corresponding maintainers $(81 \mathrm{~B}-\mathrm{a}$ common maintainer of all three lines) and eight restorers viz. H77/29-2, TCH 26-1, G 73- 107, MRC/10/61, ERC/99/35 (ERC), H77/833-2, 99HS141, HTP 92/80, selected on basis of variability for different morphological traits. Three male sterile lines and their corresponding three maintainer lines were crossed with eight restorers in line $\mathrm{x}$ tester fashion at ICRISAT, Hyderabad, during off season crop (January- April 2013).

The forty eight pearl millet hybrids, thus, produced and their parents were grown separately in contiguous blocks in two environments (two dates of sowing) $\mathrm{E}_{1}$ Planting $15^{\text {th }}$ July (Normal sowing) and $E_{2}$ Planting $24^{\text {th }}$ July (Late sowing) during kharif 2013. The experiment was raised in a Randomized Block Design with three replications in each of the environments at Research Area, CCS Haryana Agricultural University, Hisar with a plot size of 2row x 4 $\mathrm{m} \times 0.5 \mathrm{~m}$ with $10-12 \mathrm{~cm}$ intra- row spacing. All the recommended agronomic practices were followed to raise a good crop. Data was recorded on five competitive representative plants in each replication for grain yield (g/plant), dry fodder yield (g/plant), days to 50 per cent flowering, effective tillers per plant, 1000-grain weight (g), plant height $(\mathrm{cm})$, panicle length $(\mathrm{cm})$, panicle girth $(\mathrm{cm})$ and Harvest index (\%). Harvest index was calculated in percentage by the formula given by Donald (1962). Data in percentage were first subjected to angular transformation (Fisher and Yates, 1963) for analysis of variance. Combining ability analysis of 
variance was performed according to Kempthorne (1958).

\section{Results and Discussion}

The analysis of variance for combining ability (Table 1) revealed that the mean squares due to lines, testers and lines $\times$ testers were significant for all the characters in both the environments except mean squares due to lines for grain yield in $\mathrm{E}_{2}$, days to $50 \%$ flowering in $E_{2}$, panicle girth in both environments and harvest index in $\mathrm{E}_{1}$.

This revealed that lines did not show much variation for the characters in these environments. The mean squares due to testers were significant for all characters in both environments except harvest index in $\mathrm{E}_{1}$. This revealed that testers differed more for general combining ability. Highly significant mean squares due to lines $\times$ testers suggested the hybrids differed significantly for specific combining ability for all the traits in both the environments. The magnitude of mean squares due to testers was invariably higher as compared to those of lines for most of the traits like grain yield $\left(\mathrm{E}_{1}, \mathrm{E}_{2}\right)$, days o 50 per cent flowering $\left(E_{2}\right)$, Plant height $\left(E_{1}, E_{2}\right)$, panicle girth $\left(\mathrm{E}_{1}, \mathrm{E}_{2}\right)$, panicle length $\left(\mathrm{E}_{1}\right)$. This indicated that a large portion of the genetic variability in crosses was accounted by the differences in testers.

The fixed effect variances due to general combining ability (gca) and specific combining ability (sca) are presented in table 1. The magnitude of sca variances exceeded to that of gca variances for all the characters.

The ratios of gca:sca variances for all the characters less than unity (Table 1), implying preponderance of non additive gene action clearly indicting that usefulness of heterosis breeding for these traits. Preponderance of non-additive genetic control of almost all characters has been reported in a compendium of biometrical analysis in pearl millet by Virk (1988), Yadav et al., (2000), Dangaria et al., (2009), Lakshman et al., (2011) and Sumanth et al., (2013) which means the hybrids will perform better over lines and populations.

The estimates general combining ability effects of lines and testers are presented in table 2. A critical examination of gca effects of lines revealed that line $1 \quad\left(81 \mathrm{~A}_{1}\right)$ representing $\mathrm{A}_{1}$ male sterile cytoplasm proved to be good general combiner for earliness, panicle length and harvest index in one of the two environments (Table 2) and $4\left(81 B_{1}\right)$ representing $\mathrm{A}_{1}$ system fertile cytoplasm (maintainer) combined favorably for grain yield, dry fodder yield and effective tillers. The combining ability of the lines $1 / 4$ $\left(81 \mathrm{~A}_{1} / 81 \mathrm{~B}_{1}\right)$ and $2 / 5\left(81 \mathrm{~A}_{4} / 81 \mathrm{~B}_{4}\right)$ was not similar at CMS system level. Line $2\left(81 \mathrm{~A}_{4}\right)$ combined better for effective tillers and line 5 $\left(81 B_{4}\right)$ for plant height, dry fodder yield and 1000-grain weight.

The line $6\left(81 \mathrm{~B}_{5}\right)$ combined better for plant height only. Of three CMS sources along with their maintainer used in the study, $\mathrm{A}_{1}$ system $\left(\mathrm{A}_{1} / \mathrm{B}_{1}\right)$ appeared to have positive effect on many productive traits followed by $\mathrm{A}_{4}$ and A5. However, Lakshman et al., 2010 reported that $\mathrm{A}_{4}$ cytoplasm was best combiner for grain yield and other component traits followed by $A_{1}$ and $A_{5}$ cytoplasm. The results show that combining ability may be influenced for some productivity traits by type of cytoplasm and interaction with nuclear genes.

The cytoplasmic effects on combining ability were found to be modulating cytoplasmic nuclear interaction and influenced by environmental conditions (Chandra Shekara, 2007, Lakshman et al., 2010, Kumar and Sagar, 2010). 


\section{Int.J.Curr.Microbiol.App.Sci (2017) 6(8): 554-561}

Table.1 Combining ability analysis, gca and sca variances for some quantitative characters in two environments

\begin{tabular}{|c|c|c|c|c|c|c|c|c|c|c|c|c|c|c|c|c|c|c|c|}
\hline \multirow{3}{*}{$\begin{array}{c}\text { Source of } \\
\text { variation }\end{array}$} & \multirow[t]{3}{*}{ d.f. } & \multicolumn{18}{|c|}{ Mean squares } \\
\hline & & \multicolumn{2}{|c|}{$\begin{array}{c}\text { Grain yield } \\
\text { (g/plant) }\end{array}$} & \multicolumn{2}{|c|}{$\begin{array}{l}\text { Dry fodder yield } \\
\text { (g/plant) }\end{array}$} & \multicolumn{2}{|c|}{$\begin{array}{c}\text { Days to } 50 \% \\
\text { flowering }\end{array}$} & \multicolumn{2}{|c|}{ Plant height $(\mathrm{cm})$} & \multicolumn{2}{|c|}{$\begin{array}{l}\text { Effective tillers } \\
\text { (No./plant) }\end{array}$} & \multicolumn{2}{|c|}{$\begin{array}{l}\text { 1000-grain } \\
\text { weight }(\mathrm{g})\end{array}$} & \multicolumn{2}{|c|}{$\begin{array}{l}\text { Panicle girth } \\
\text { (cm) }\end{array}$} & \multicolumn{2}{|c|}{$\begin{array}{l}\text { Panicle length } \\
(\mathrm{cm})\end{array}$} & \multicolumn{2}{|c|}{$\begin{array}{c}\text { Harvest Index } \\
(\%)\end{array}$} \\
\hline & & $\mathbf{E}_{1}$ & $\mathbf{E}_{2}$ & $\mathbf{E}_{1}$ & $\mathbf{E}_{2}$ & $\mathbf{E}_{1}$ & $\mathbf{E}_{2}$ & $\mathbf{E}_{1}$ & $\mathbf{E}_{2}$ & $\overline{\mathbf{E}_{1}}$ & $\mathbf{E}_{2}$ & $\mathbf{E}_{1}$ & $\overline{\mathbf{E}_{2}}$ & $\mathbf{E}_{1}$ & $\mathbf{E}_{2}$ & $\overline{\mathbf{E}_{1}}$ & $\mathbf{E}_{2}$ & $\overline{\mathbf{E}_{1}}$ & $\mathbf{E}_{2}$ \\
\hline Replication & 2 & 7.29 & 0.2 & 25.22 & 219.32 & 13.22 & 10.7 & 592.22 & 98.1 & 0.01 & 0.05 & 0.47 & 0.03 & 0.005 & 0.04 & 16.08 & 1.63 & 4.63 & 5.78 \\
\hline Hybrids & 47 & $35.86^{* *}$ & $29.47 * *$ & $392.66^{* *}$ & $413.10^{* *}$ & $64.53 * *$ & $14.62 * *$ & $757.64 * *$ & $677.61 * *$ & $0.74 * *$ & $0.50 * *$ & $2.50 * *$ & $1.87 * *$ & $0.38 * *$ & $0.37 * *$ & $13.25 * *$ & $\begin{array}{c}14.76 \\
* *\end{array}$ & $9.40 * *$ & $\begin{array}{c}21.95 \\
* *\end{array}$ \\
\hline Lines & 5 & $32.14 * *$ & 18.18 & $590.03 * *$ & $627.42 * *$ & $77.67 * *$ & 1.93 & $1147.55 * *$ & $293.21 * *$ & $0.99 * *$ & $0.54 * *$ & $1.77 * *$ & $1.15 * *$ & 0.1 & 0.09 & $10.00 * *$ & $\begin{array}{c}18.18 \\
* *\end{array}$ & 8.68 & $\begin{array}{c}34.59 \\
* *\end{array}$ \\
\hline Testers & 7 & $32.91 * *$ & $37.42 * *$ & $338.07^{* * *}$ & $307.15^{* *}$ & $21.01 * *$ & $30.74 * *$ & $2125.80 * *$ & $343.16^{* *}$ & $0.86^{* *}$ & $0.42 * *$ & $2.06^{* *}$ & 1.05 & $0.54 * *$ & $0.76 * *$ & $21.39 * *$ & $\begin{array}{c}8.60 * \\
*\end{array}$ & 6.35 & $\begin{array}{c}9.18 * \\
*\end{array}$ \\
\hline $\begin{array}{l}\text { Line } \mathrm{x} \\
\text { Testers }\end{array}$ & 35 & $36.98 * *$ & $29.48 * *$ & $375.38 * *$ & $403.66 * *$ & $71.35^{* *}$ & $13.21 * *$ & $428.33 * *$ & $799.45^{* *}$ & $0.68 * *$ & $0.52 * *$ & $2.69 * *$ & $2.14 * *$ & $0.39 * *$ & 0.34 & $12.09 * *$ & $\begin{array}{c}15.50 \\
* *\end{array}$ & $10.11 * *$ & $\begin{array}{c}22.70 \\
* *\end{array}$ \\
\hline Error & 94 & 9.95 & 9.72 & 52.24 & 42.82 & 5.2 & 2.97 & 92.99 & 78.03 & 0.1 & 0.06 & 0.4 & 0.17 & 0.05 & 0.05 & 1.8 & 1.83 & 4.04 & 3.74 \\
\hline $\begin{array}{c}\sigma^{2} \text { gca } \\
\text { variances }\end{array}$ & & -0.21 & -0.08 & 4.22 & 3.03 & -1.04 & 0.14 & 57.54 & -22.91 & 0.01 & -0.002 & -0.03 & -0.05 & -0.003 & 0.004 & 0.17 & -0.10 & -0.12 & -0.03 \\
\hline $\begin{array}{c}\sigma^{2} \text { sca } \\
\text { variances }\end{array}$ & & 9.00 & 6.588 & 107.71 & 120.28 & 22.04 & 3.41 & 111.77 & 240.47 & 0.19 & 0.15 & 0.76 & 0.65 & 0.11 & 0.09 & 3.42 & 4.55 & 2.02 & 6.32 \\
\hline $\begin{array}{c}\text { Ratio } \\
\sigma^{2} \text { gca } \\
/ \sigma^{2} \text { sca } \\
\text { variances }\end{array}$ & & -0.02 & -0.01 & 0.04 & 0.03 & -0.05 & 0.04 & 0.52 & -0.09 & 0.05 & -0.01 & -0.001 & -0.08 & -0.03 & 0.04 & 0.05 & -0.02 & -0.06 & -0.01 \\
\hline
\end{tabular}




\section{Int.J.Curr.Microbiol.App.Sci (2017) 6(8): 554-561}

Table.2 Estimates of general combining ability effects of lines and testers for different characters in two environments

\begin{tabular}{|c|c|c|c|c|c|c|c|c|c|c|c|c|c|c|c|c|c|c|c|}
\hline \multirow[t]{2}{*}{ S. No. } & \multirow[t]{2}{*}{ Lines } & \multicolumn{2}{|c|}{$\begin{array}{l}\text { Grain yield } \\
\text { (g/plant) }\end{array}$} & \multicolumn{2}{|c|}{$\begin{array}{l}\text { Dry fodder yield } \\
\text { (g/plant) }\end{array}$} & \multicolumn{2}{|c|}{$\begin{array}{l}\text { Days to } 50 \% \\
\text { flowering }\end{array}$} & \multicolumn{2}{|c|}{ Plant height $(\mathrm{cm})$} & \multicolumn{2}{|c|}{$\begin{array}{l}\text { Effective tillers } \\
\text { (No./plant) }\end{array}$} & \multicolumn{2}{|c|}{$\begin{array}{l}\text { 1000-grain } \\
\text { weight (g) }\end{array}$} & \multicolumn{2}{|c|}{$\begin{array}{l}\text { Panicle girth } \\
\quad(\mathrm{cm})\end{array}$} & \multicolumn{2}{|c|}{$\begin{array}{l}\text { Panicle length } \\
(\mathrm{cm})\end{array}$} & \multicolumn{2}{|c|}{$\begin{array}{l}\text { Harvest index } \\
\qquad(\%)\end{array}$} \\
\hline & & $\mathrm{E}_{1}$ & $\mathrm{E}_{2}$ & $\mathrm{E}_{1}$ & $\mathrm{E}_{2}$ & $\overline{E_{1}}$ & $\mathrm{E}_{2}$ & $\overline{E_{1}}$ & $\mathrm{E}_{2}$ & $\overline{E_{1}}$ & $\overline{\mathrm{E}_{2}}$ & $\overline{E_{1}}$ & $\mathrm{E}_{2}$ & $\mathrm{E}_{1}$ & $\mathrm{E}_{2}$ & $\mathrm{E}_{1}$ & $\overline{E_{2}}$ & $\mathrm{E}_{1}$ & $\mathrm{E}_{2}$ \\
\hline 1 & $81 \mathrm{~A}_{1}$ & 0.42 & 1.38 & 0.85 & -2.86 & $-1.79^{*}$ & 0.2 & 1.24 & 1.3 & -0.07 & 0.08 & -0.01 & 0.20 & 0.01 & -0.06 & -0.12 & $1.23^{*}$ & -0.00 & $1.34^{*}$ \\
\hline 2 & $81 \mathrm{~A}_{4}$ & -1.72 & -0.16 & $-5.05^{*}$ & -0.58 & $1.71^{*}$ & -0.49 & 0.36 & -0.97 & $0.24^{*}$ & $0.21 *$ & 0.12 & 0.02 & -0.01 & 0.07 & -0.14 & -0.09 & 0.12 & -0.10 \\
\hline 3 & $81 \mathrm{~A}_{5}$ & -0.21 & 0.57 & -3.41 & $-4.35 *$ & -0.54 & -0.02 & $-8.49 *$ & -3.82 & -0.17 & -0.12 & 0.29 & -0.02 & -0.12 & -0.05 & $-1.12 *$ & -0.74 & 0.66 & 0.92 \\
\hline 4 & $81 \mathrm{~B}_{1}$ & $1.83^{*}$ & -1.12 & $5.61^{*}$ & $10.00^{*}$ & -1.28 & 0.001 & $-5.95 *$ & -1.99 & $0.25^{*}$ & 0.04 & $-0.45 *$ & $-0.32 *$ & 0.07 & -0.03 & 0.66 & 0.69 & -0.20 & $-2.12^{*}$ \\
\hline 5 & $81 B_{4}$ & 0.07 & -0.37 & $6.07^{*}$ & -0.53 & $2.72^{*}$ & 0.33 & 1.53 & $6.25^{*}$ & -0.04 & -0.02 & 0.19 & $0.27 *$ & 0.05 & 0.08 & 0.19 & $-1.10^{*}$ & -1.0 & -0.13 \\
\hline 6 & $81 \mathrm{~B}_{5}$ & $\begin{array}{l}-0.39 \\
-0.3\end{array}$ & $\begin{array}{l}-0.29 \\
\end{array}$ & -4.06 & -1.66 & -0.8 & $\begin{array}{l}-0.01 \\
\end{array}$ & $11.29^{*}$ & $\begin{array}{l}-0.76 \\
\end{array}$ & $-0.20 *$ & $-0.20^{*}$ & -0.14 & $\begin{array}{l}-0.14 \\
\end{array}$ & $\begin{array}{c}-0.01 \\
\end{array}$ & $\begin{array}{l}-0.01 \\
\end{array}$ & 0.54 & 0.01 & 0.47 & 0.09 \\
\hline $\begin{array}{l}\text { C.D. at } \\
-\mathrm{P} 0.05\end{array}$ & & 1.81 & 1.79 & 4.13 & 3.74 & 1.29 & 0.97 & 5.53 & 5.07 & 0.18 & 0.13 & 0.35 & 0.23 & 0.13 & 0.13 & 0.75 & 0.77 & 1.15 & 1.09 \\
\hline & Testers & & & & & & & & & & & & & & & & & & \\
\hline 7 & $\mathrm{H} 77 / 29-2$ & 1.58 & 0.001 & $5.07 *$ & $\begin{array}{l}-3.87 \\
\end{array}$ & 0.44 & $\begin{array}{l}-0.42 \\
\end{array}$ & 4.26 & 2.75 & 0.16 & 0.03 & $0.72 *$ & 0.22 & $-0.24^{*}$ & $-0.21^{*}$ & $\begin{array}{l}-0.71 \\
\end{array}$ & 0.01 & -0.10 & 0.84 \\
\hline 8 & TCH 26-1 & 1.34 & 0.09 & -3.18 & -3.02 & 0.44 & 0.41 & 6.37 & 1.17 & $0.24 *$ & -0.09 & -0.37 & 0.19 & -0.04 & 0.01 & $1.57^{*}$ & -0.36 & 0.98 & 0.41 \\
\hline 9 & G 73-107 & -0.79 & -0.52 & 0.87 & 1.85 & -0.48 & 0.21 & 6.17 & -0.04 & 0.17 & $0.30^{*}$ & -0.36 & $-0.27^{*}$ & $0.22 *$ & 0.03 & $-0.94^{*}$ & $-1.35^{*}$ & -0.6 & -0.53 \\
\hline 10 & MRC & 1.52 & 1.24 & 3.86 & -3.65 & $1.83^{*}$ & $1.60^{*}$ & -2.44 & $-7.78 *$ & -0.21 & 0.09 & -0.06 & -0.1 & -0.05 & $0.28 *$ & -0.21 & -0.22 & 0.24 & 1.08 \\
\hline 11 & ERC & -1.01 & $-2.61 *$ & $-5.26 *$ & -3.21 & -0.18 & -0.2 & 3.9 & 4.51 & -0.08 & 0.03 & 0.01 & $0.38^{*}$ & 0.03 & $\begin{array}{l}-0.11 \\
\end{array}$ & -0.71 & 0.82 & 0.58 & $\begin{array}{l}-0.89 \\
\end{array}$ \\
\hline 12 & $\begin{array}{c}\mathrm{H} 77 / 833- \\
2\end{array}$ & -0.75 & 0.001 & 1.87 & 2.54 & 0.61 & 0.41 & $7.98^{*}$ & 5.16 & 0.2 & -0.11 & 0.04 & -0.17 & $-0.19 *$ & $-0.23 *$ & $-1.18^{*}$ & 0.3 & -0.7 & -0.31 \\
\hline 13 & 99HS141 & 0.09 & -0.57 & 3.04 & 1.7 & -1.02 & $-2.84 *$ & $-25.37 *$ & -3.14 & $-0.25 *$ & -0.1 & 0.04 & -0.23 & $0.23^{*}$ & $0.30^{*}$ & 0.85 & 0.73 & -0.5 & -0.57 \\
\hline 14 & $\begin{array}{c}\text { HTP } \\
92 / 80\end{array}$ & -1.98 & $2.36^{*}$ & $-6.27 *$ & $7.66^{*}$ & $-1.64 *$ & 0.82 & -0.88 & -2.63 & $-0.24 *$ & -0.16 & -0.01 & -0.01 & 0.05 & -0.08 & $1.32^{*}$ & 0.06 & 0.1 & -0.03 \\
\hline $\begin{array}{l}\text { C.D. at } \\
=\text { P } 0.05\end{array}$ & & 2.08 & 2.06 & 4.77 & 4.33 & 1.51 & 1.13 & 6.38 & 5.85 & 0.21 & 0.16 & 0.41 & 0.27 & 0.13 & 0.15 & 0.87 & 0.89 & 1.33 & 1.27 \\
\hline
\end{tabular}


Table.3 Specific combining ability effects and per se performance (in parentheses) for top five hybrids for some quantitative characters in two environments during 2013 season

\begin{tabular}{|c|c|c|c|c|c|}
\hline \multirow[t]{2}{*}{ Character } & \multirow[t]{2}{*}{ Cross } & \multicolumn{2}{|c|}{ Environments } & \multicolumn{2}{|c|}{ gca status of parents } \\
\hline & & $E_{1}$ & $\mathrm{E}_{2}$ & $E_{1}$ & $\mathrm{E}_{2}$ \\
\hline \multirow[t]{2}{*}{ Grain yield $(\mathrm{g} / \mathrm{plant}$} & $3 \times 11$ & $3.89(28.91)$ & $5.45 *(28.05)$ & - & Average $\mathrm{x}$ Poor \\
\hline & $4 \times 8$ & $6.33 *(35.73)$ & $-2.11(21.44)$ & Good x Average & - \\
\hline \multirow[t]{5}{*}{ Dry fodder yield (g/plant) } & $3 \times 9$ & $12.77 *(84.33)$ & $12.45^{*}(89.33)$ & Average $x$ Average & Good x Average \\
\hline & $3 \times 13$ & $17.23 *(90.97)$ & $11.80 *(88.53)$ & Average $\mathrm{x}$ Poor & Poor x Poor \\
\hline & $4 \times 8$ & $12.21 *(88.73)$ & $1.97(88.33)$ & Good x Average & Good x Average \\
\hline & $5 \times 14$ & $14.43 *(88.33)$ & $5.29(91.80)$ & Good x Poor & Average x Good \\
\hline & $1 \times 9$ & $-11.36(64.47)$ & $11.63 *(90.00)$ & - & Average $\mathrm{x}$ Average \\
\hline \multirow[t]{5}{*}{ Days to $50 \%$ flowering } & $1 \times 7$ & $-4.48 *(47.17)$ & $-1.75(45.50)$ & Good x Average & - \\
\hline & $1 \times 9$ & $-6.25 *(44.47)$ & $-2.39(45.50)$ & Good x Average & - \\
\hline & $2 \times 9$ & $-4.56 *(49.67)$ & $0.97(48.17)$ & Poor x Average & - \\
\hline & $3 \times 8$ & $-7.56 *(45.33)$ & $2.70(45.17)$ & Average $\mathrm{x}$ Average & - \\
\hline & $4 \times 13$ & $-6.51 *(44.17)$ & $-3.96 *(40.67)$ & Average x Average & Average x Good \\
\hline \multirow[t]{5}{*}{ Plant height $(\mathrm{cm})$} & $1 \times 9$ & $7.72(180.27)$ & $23.79 *(178.17)$ & - & Average $\mathrm{x}$ Average \\
\hline & $1 \times 10$ & $-2.91(161.00)$ & $17.06 *(163.70)$ & - & Average x Poor \\
\hline & $2 \times 7$ & $14.98(184.73)$ & $26.30 *(181.20)$ & - & Average x Average \\
\hline & $3 \times 8$ & $10.20(173.20)$ & $25.30 *(175.77)$ & - & Average $\mathrm{x}$ Average \\
\hline & $6 \times 13$ & $28.76 *(179.80)$ & $22.59 *(171.80)$ & Good x Poor & Average $\mathrm{x}$ Average \\
\hline \multirow[t]{5}{*}{ Effective tillers (No./plant) } & $1 \times 10$ & $-0.20(2.13)$ & $0.68 *(3.20)$ & - & Average $\mathrm{x}$ Average \\
\hline & $2 \times 13$ & $1.10 *(3.73)$ & $0.6 *(3.13)$ & Good x Poor & Good x Average \\
\hline & $3 \times 11$ & $0.70 *(3.07)$ & $0.56 *(2.80)$ & Average $\mathrm{x}$ Poor & Average $\mathrm{x}$ Average \\
\hline & $4 \times 12$ & $0.77 *(3.87)$ & $0.46 *(2.73)$ & Good x Average & Average $\mathrm{x}$ Average \\
\hline & $1 \times 9$ & $0.27(3.00)$ & $0.73 *(3.47)$ & - & Average x Good \\
\hline \multirow[t]{5}{*}{ 1000- grain weight $(\mathrm{g})$} & $1 \times 11$ & $1.50 *(8.33)$ & $1.86 *(9.30)$ & Average x Average & Average x Good \\
\hline & $1 \times 13$ & $1.80 *(8.67)$ & $1.52 *(8.33)$ & Average x Average & Average $\mathrm{x}$ Average \\
\hline & $2 \times 10$ & $1.81 *(8.70)$ & $1.62 *(8.40)$ & Average x Average & Average $\mathrm{x}$ Average \\
\hline & $3 \times 7$ & $1.46^{*}(9.30)$ & $1.08 *(8.13)$ & Average $\mathrm{x}$ Average & Average $\mathrm{x}$ Average \\
\hline & $3 \times 14$ & $1.56 *(8.67)$ & $1.44 *(8.27)$ & Average x Good & Average $\mathrm{x}$ Average \\
\hline \multirow[t]{5}{*}{ Panicle girth $(\mathrm{cm})$} & $3 \times 11$ & $0.68 *(2.77)$ & $0.74 *(2.53)$ & Average x Average & Average $\mathrm{x}$ Average \\
\hline & $5 \times 8$ & $0.71 *(2.90)$ & $0.60 *(2.67)$ & Average $\mathrm{x}$ Average & Average $\mathrm{x}$ Average \\
\hline & $2 \times 14$ & $0.50 *(2.72)$ & $0.58 *(2.53)$ & Average $\mathrm{x}$ Average & Average $\mathrm{x}$ Average \\
\hline & $6 \times 10$ & $0.38 *(2.50)$ & $0.48 *(2.73)$ & Average x Average & Average x Good \\
\hline & $1 \times 10$ & $0.66 *(2.80)$ & $0.38(2.56)$ & Average $\mathrm{x}$ Average & Average x Good \\
\hline \multirow[t]{5}{*}{ Panicle length $(\mathrm{cm})$} & $1 \times 10$ & $3.86 *(24.27)$ & $2.86 *(22.33)$ & Average $\mathrm{x}$ Average & Good x Average \\
\hline & $2 \times 7$ & $2.55 *(22.46)$ & $3.05 *(21.43)$ & Average $\mathrm{x}$ Average & Average $\mathrm{x}$ Average \\
\hline & $4 \times 12$ & $2.27 *(22.50)$ & $3.86 *(23.33)$ & Average $\mathrm{x}$ Poor & Average $\mathrm{x}$ Average \\
\hline & $4 \times 8$ & $4.88 *(27.88)$ & $-0.13(18.67)$ & Average x Good & - \\
\hline & $5 \times 10$ & $1.98(22.70)$ & $3.67 *(20.80)$ & - & Average x Average \\
\hline \multirow[t]{5}{*}{ Harvest index (\%) } & $1 \times 7$ & $\begin{array}{c}2.27(29.64) \\
\mathbf{( 3 2 . 9 6 )}\end{array}$ & $\begin{array}{c}5.73 *(36.46) \\
(\mathbf{3 7 . 1 2}) \\
\end{array}$ & - & Good x Average \\
\hline & $6 \times 9$ & $\begin{array}{c}1.52(28.45) \\
(\mathbf{3 2 . 1 6})\end{array}$ & $\begin{array}{c}3.52 *(28.58) \\
(\mathbf{3 2 . 3 0})\end{array}$ & - & Average x Average \\
\hline & $2 \times 12$ & $\begin{array}{c}1.74(28.29) \\
(\mathbf{3 2 . 0 2}) \\
\end{array}$ & $\begin{array}{c}4.77 *(30.57) \\
(\mathbf{3 3 . 5 4}) \\
\end{array}$ & - & Average x Average \\
\hline & $1 \times 10$ & $\begin{array}{c}-1.14(24.84) \\
(\mathbf{2 9 . 8 7 )}\end{array}$ & $\begin{array}{c}3.79 *(33.71) \\
(\mathbf{3 5 . 4 6 )}\end{array}$ & - & Good x Average \\
\hline & $3 \times 11$ & $\begin{array}{c}2.19(31.85) \\
(\mathbf{3 4 . 3 0 )}\end{array}$ & $\begin{array}{c}4.53 *(30.90) \\
(\mathbf{3 3 . 7 6})\end{array}$ & - & Average x Average \\
\hline
\end{tabular}

* significant at $\mathrm{P}=0.05$

Bold figures in parentheses are angular transformed values; $E_{1}$ and $E_{2}=$ Normal and late sown crops, respectively 
Among testers, significant high gca effects were recorded for grain yield - 14 (HTP 92/80), dry fodder yield - 7 (H77/29-2), days to 50 per cent flowering - 13 (99HS141) and 14 (HTP 92/80), plant height- 12 (H77/8332), effective tillers- 8 (TCH 26-1), 9 (G73107), 1000-grain weight- 7 (H 77/29-2) and 11 (ERC), panicle girth- 13 (99HS141), 9 (G73-107) and 10 (MRC), panicle length- 8 (TCH 26-1) and 14 (HTP 92/80). The tester 14 (HTP 92/80) turned to be good general combiner in one or both the environments for grain yield and some other traits such as dry fodder yield, earliness and panicle length.

A list of few selected crosses on the basis of sca effects for various characters, per se and gca status of parents is given in table 3 . The crosses in general did not express significant high sca effects in both the environments for all the characters studied. Kumar (2002) also reported similar observations. The hybrid $3 \times 11\left(81 \mathrm{~A}_{5} \times \mathrm{ERC}\right)$ involving average and poor combiners for grain yield expressed significant positive sca effects in one of the two environments and also gave high per se performance. This hybrid also combined favorably for effective tillers, panicle girth and harvest index (positive sca effects), therefore, this hybrid needs further vigorous testing. The cross $1 \times 7 \quad\left(81 \mathrm{~A}_{1} \times \mathrm{H} 77 / 29-2\right)$ proved to be desirable for days to $50 \%$ flowering and harvest index. The cross $1 \times 9$ $\left(81 A_{1} \times\right.$ G73-107) proved to be desirable for dry fodder yield, days to $50 \%$ flowering, plant height and effective tillers. The cross $1 \times 10$ $\left(81 \mathrm{~A}_{1} \times \mathrm{MRC}\right)$ proved to be desirable for plant height, effective tillers, panicle girth, panicle length and harvest index.

None of the hybrids involving goodx good combining parents gave high sca effects and high per se performance. The hybrid $4 \times 8$ $\left(81 \mathrm{~B}_{1} \times \mathrm{TCH} 26-1\right)$ involving good $\times$ average combining parents gave positive sca effects in at least one environment for grain yield.
Besides grain yield, it also gave high sca and per se performance for dry fodder yield and panicle length in one of the two environments. It is not essential that both parents having good combining ability will give high SCA as also reported by Sagar (1990) and Kumar (2002). Further, the $A_{5}$ cytoplasmic male sterility system lines and pollinators such as ERC, HTP 92/80 and TCH 26-1 and G73-107 can be used in breeding for high yielding hybrids. These pollinators can also be crossed with newly developed male sterile lines. The hybrid $3 \times 11 \quad\left(81 \mathrm{~A}_{5} \times \mathrm{ERC}\right)$ needs further vigorous testing. The study also indicated that in addition to the widely used $\mathrm{A}_{1}$ CMS source, $\mathrm{A}_{5}$ CMS source offered potential alternative to diversify the male sterile cytoplasm in pearl millet crop.

\section{Acknowledgements}

Studentship support, provided by the CCS Haryana Agricultural University, Hisar to conduct present work as part of M Sc thesis, is duly acknowledged. We express our sincere thanks to Dr. Prem Sagar, Ex- Professor and Head, Bajra Section, Department of Genetics and plant breeding for comments and suggestions.

\section{References}

Aken'ova, M.E. 1985. Confirmation of a new source of cytoplasmic-genic male sterility in bullrush millet [Pennisetum typhoides (L.) Leeke.]. Euphytica, 34: 34-38.

Anonymous. 2016. Agricultural statistics at a glance. Ministry of Agriculture, Government of India, New Delhi.

Appadurai, R., Raveendran, T.S., and Nagarajan, C. 1982. A new male sterility system in pearl millet. Indian J. Agric. Sci., 52: 832834.

Athwal, D.S. 1965. Hybrid bajra-1 marks a new era. Indian Farming, 15(5): 6-7.

Burton, G.W. 1958. Cytoplasmic male-sterility in pearl millet [Pennisetum glaucum (L.) R. Br.]. Agron. J., 50: 230-231. 
Burton, G.W. 1965. Pearl millet Tift 23A released. Crops and Soils, 17: 19.

Burton, G.W., and Athwal, D.S. 1967. Two additional sources of cytoplasmic malesterility in pearl millet and their relationship to Tift 23A. Crop Sci., 7: 209-211.

Dangariya, C.J., Chotalia, J.M., Savaliya, J.J., Davda, B.K., and Pansuriya, A.G. 2009. Combining ability analysis in pearl millet [Pennisetum glaucum (L.) R. Br.]. Agric. Sci. Digest., 29(4): 287-290.

Donald, C.M. 1962. In search of yield. $J$. Australian Institute Agric. Sci., 28: 171178.

Fisher, R.A., and Yates, F. 1963. Statistical Table for Biological, Agricultural and Medical Research, Oliver and Boyd, London.

Hanna, W.W. 1989. Characteristics and stability of a new cytoplasmic-nuclear male-sterile source in pearl millet. Crop Sci., 29: 14571459.

Kempthorne, O. 1957. An introduction to genetic statistics John Wiley and Sons, New York, USA.

Kumar, R. 2002. Studies on effect of cytoplasm on productive traits and combining ability in direct sown and regenerated pearl millet [Pennisetum glaucum (L.) R. BR.]. Ph.d. Thesis, Haryana Agricultural University, Hisar.

Kumar, R., and Sagar, P. 2010. Effect of cytoplasm on combining ability and yield attributes in pearl millet [Pennisetum glaucum (L.) R. Br.]. Indian J. Genet., 70(3): 247-256.

Lakshmana, D., Biradar, B.D., Deshpande, S.K., and Madaiah, D. 2010. Studies on combining ability and heterosis involving diverse cytoplasmic male sterility system in pearl millet. Indian J. Genet., 70(1): 29-33. Lakshmana, D., Biradar, B.D., Madaiah, D., and Jolli, R.B. 2011. Combining ability studies on $A_{1}$ source of cytoplasmic male sterility system in pearl millet. Indian J. Agric. Res., 45(1): 45-51.

Marchais, L., and Pernes, J. 1985. Genetic divergence between wild and cultivated pearl millet (Pennisetum typhoides) 1. male sterility. Z. Pflanzenziichtung, 95: 103-112.

NIN. 2003. Nutritive value of Indian Foods. Ed Gopalan and Deosthale, National Institute of Nutrition, Hyderabad.

Panse, V.G., and Sukhatme, P.V. 1967. "Statistical methods for agricultural workers". ICAR, New Delhi.

Rai, K.N. 1995. A new cytoplasmic-nuclear male sterility system in pearl millet. Plant Breed, 114: 445-447.

Sagar, P. 1990. Inheritance of plant height in pearl millet. Indian J. Genet., 50(3): 233-239.

Sumanth, M., Sumathi, P., Vinodhana, N.K., and Sathya, M. 2013. Assessment of genetic distance among the inbred lines of pearl millet [Pennisetum glaucum (L.) R. BR] using SSR markers. Int. J. Biotech. Allied Fields, 1(3): 153-162.

Virk, D.S. 1988. Biometrical analysis in pearl millet - a review. Crop Improv., 15(1): 129.

Yadav, O.P., Weltzien, E.R., Mahalakhmi, V., and Bidinger, F.R. 2000. Combining ability of pearl millet landraces originating from arid area of Rajasthan. Indian J. Genet., 60(1): 45-53.

Young, J.B., and Virmani, S.S. 1990. Effects of cytoplasm on heterosis and combining ability for agronomic traits in rice (Oryza sativa L.). Euphytica, 48: 177-188.

\section{How to cite this article:}

Anil Kumar, R. Kumar, Devvart and A.K. Dehinwal. 2017. Combining Ability for Grain Yield and its Components Involving Alloplasmic Iso-Nuclear Lines of Pearl Millet. Int.J.Curr.Microbiol.App.Sci. 6(8): 554-561. doi: https://doi.org/10.20546/ijcmas.2017.608.072 